\title{
Multispectral enhancement towards digital staining
}

\author{
Pinky A. Bautista* and Yukako Yagi \\ Department of Pathology, Massachusetts General Hospital, Harvard Medical School, Boston, MA, USA
}

\begin{abstract}
Background: Digital staining can be considered as a special form of image enhancement wherein the concern is not only to increase the contrast between the background objects and objects of interest, but to also impart the colors that mark the objects' unique reactions to a specific stain. In this paper, we extended the previously proposed multispectral enhancement methods such that the colors of the background pixels can also be changed.

Methods: In the previous multispectral enhancement methods a shifting factor is provided to the original spectrum. To implement digital staining, a spectral transformation process is introduced prior to spectral shifting.

Results: The enhancement method is applied to multispectral images of H\&E stained liver tissue. The resulting digitally stained images show good correlation with the serial-section images of the tissue which were physically stained with Masson's trichrome.

Conclusions: We have presented a multispectral enhancement method that can be adjusted to produce digitally stainedimages. The current experimental results show the viability of the method. However, to achieve robust enhancement performance issues that arise from variations in staining conditions has to be addressed as well. This would be part of our future work.
\end{abstract}

Keywords: Multispectral imaging, multispectral enhancement, spectral enhancement, digital staining, digital pathology

\section{Introduction}

Staining of tissue slides is an important process in pathology diagnosis workflow. The colors acquired by the tissue structures from staining are valuable to their classification. For instance, from a hematoxylin and eosin (H\&E) stained tissue slides, cytoplasm and connective tissues are stained pink to red while the nuclei are stained blue to dark blue. Aside from H\&E stain, which is generally used for routine staining, special stains, such as the Masson's trichrome stain, are also used to further highlight the differences between tissue structures which acquired similar H\&E staining patterns, i.e., collagen fiber and smooth muscle. Masson's trichrome stain is typically used in conjunction

${ }^{*}$ Corresponding author: Dr. Pinky A. Bautista, Department of Pathology, Massachusetts General Hospital, Harvard Medical School, Boston, MA 02114, USA. E-mail: pbautista@partners.org. with H\&E stain to have better visualization of the collagen fiber regions [1]. With this stain, the collagen fibers appear blue while the nuclei brown to black and the muscle fibers and cytoplasm pink to red.

The digital conversion of a multispectral $\mathrm{H} \& \mathrm{E}$ stained image into Masson's trichrome stained image was investigated in [2]. The method consisted of two steps: (i) classification of the image pixels using spectral features; and (ii) spectral transformation. The spectral transformation of an H\&E stained spectrum to its Masson's trichrome stained spectrum equivalent is performed using an $N x N$ transformation matrix, $N=$ total number of multispectral bands, which is calculated by least mean square method using the $H \& E$ and Masson's trichrome spectral data set of the different tissue components. The use of spectral enhancement together with spectral transformation as an approach for digital staining is recently proposed [3]. The utilization of spectral enhancement enables the 
implementation of digital staining without necessarily performing spectral classification and using multiple transformation matrices.

Digital staining results to an enhanced image wherein the objects of interest and the background objects are shaded with colors associated to their chemical staining patterns. While the proposed multispectral image enhancement method in [4] has the capability to digitally color the H\&E stained collagen fibers with colors associated to their Masson's trichrome staining patterns, it doesn't have the capability to modify the color of the background objects according to the desired staining impression. In this paper, we extended the enhancement method in [4] such that the colors of both the background and object of interest pixels could be changed.

\section{Materials and methods}

\subsection{Tissue images}

Two tissue slides belonging to the serial sections of a liver specimen were respectively stained with $H \& E$ and Masson's trichrome. Five pairs of H\&E and Masson's trichrome stained multispectral images were captured at an optical magnification of $20 \times$ using the Olympus microscope which is fitted with a multispectral filter whose sensitivity spans the visible spectrum, i.e., $400-720 \mathrm{~nm}$. Each multispectral image is a composite of $N$-grey level images which illustrate the spectral sensitivities of the stained tissue structures to light at different wavelengths.

\subsection{Multispectral enhancement}

The multispectral enhancement proposed in [4] was based on the method proposed by Mitsui et al. [5] which can be expressed as follows:

$$
\mathbf{t}_{\mathrm{e}}=\mathbf{t}_{\mathrm{o}}+\mathbf{W \varepsilon}
$$

where, $\mathbf{t}_{\mathrm{o}}, \mathbf{t}_{\mathrm{e}}$ and $\boldsymbol{\varepsilon}$ are all $N \times 1$ column vectors. The notations $\mathbf{t}_{\mathrm{o}}$ and $\mathbf{t}_{\mathrm{e}}$ represent the original and enhanced $\mathrm{N}$-band spectral transmittance of the multispectral pixel, while represents the spectral-residual error. The spectral residual error is defined as the difference between the pixel's original spectral transmittance, $\mathbf{t}_{\mathrm{o}}$, and the pixel's estimated transmittance $\mathbf{t}^{\prime}$ :

$$
\begin{aligned}
\boldsymbol{\varepsilon} & =\mathbf{t}_{\mathrm{o}}-\mathbf{t}^{\prime} \\
\mathbf{t}^{\prime} & =\sum_{i=1}^{m} \alpha_{i} \mathbf{v}_{i}+\overline{\mathbf{t}}
\end{aligned}
$$

where $\alpha_{i}$ and $\mathbf{v}_{i}$ denote the $i$ th principal component (PC) coefficient and PC vector, respectively; and $\overline{\mathbf{t}}$, represents the average spectral transmittance of the background pixels. The PC vectors $\mathbf{v}$ and the average spectral transmittance $\overline{\mathbf{t}}$ are calculated from the spectral transmittance of the background pixels; we refer to the background pixels as the pixels which do not belong to the objects of interest. The desired color for enhancement can be achieved by designing the $N \times N$ weighting matrix $\mathbf{W}$ appropriately. The authors in [4] showed that this can be done by defining the $q$ th column of the matrix $\mathbf{W}$ as follows:

$$
[\mathbf{W}]_{q}=\left\{\begin{array}{l}
k\left(\mathbf{t}_{\mathrm{o}}-\mathbf{t}_{\mathrm{a}}\right) q=n \\
0 \quad \text { otherwise }
\end{array}\right.
$$

where $\mathbf{t}_{\mathrm{a}}$ denotes the average spectral transmittance of all the pixels in the image. The parameter $k$ is a real number, which function as a scaling factor to the difference between the original transmittance, $\mathbf{t}_{\mathrm{o}}$, and average transmittance $t_{a}$. The band for enhancement, $n$, is associated to the band at which the spectral residual-error, $\boldsymbol{\varepsilon}$, of the object of interest, e.g, collagen fiber, peaks while the error of the rest of the tissue components tapers to zero. In the present work we made a modification to the definition of the weighting matrix $\mathrm{W}$ as follows:

$$
[\mathbf{W}]_{q}=\left\{\begin{array}{l}
k\left(\mathbf{t}_{\mathrm{d}}-\mathbf{t}_{\mathrm{o}}\right) q=n \\
0 \quad \text { otherwise }
\end{array}\right.
$$

We replaced $\mathbf{t}_{\mathrm{a}}$ with the original transmittance of the pixel, $\mathbf{t}_{0}$, such that the $q$ th column components of the weighting matrix $\mathbf{W}$ are unique for every pixel. By defining the components of the matrix $\mathbf{W}$ this way, the lightness of the enhanced image will not be necessarily affected when the hues of the background pixels are not similar.

\subsection{Multispectral enhancement and digital staining}

We extended the multispectral formulation in eqn.1 to provide an option to also change the colors of the background pixels by introducing an $N \times N$ 


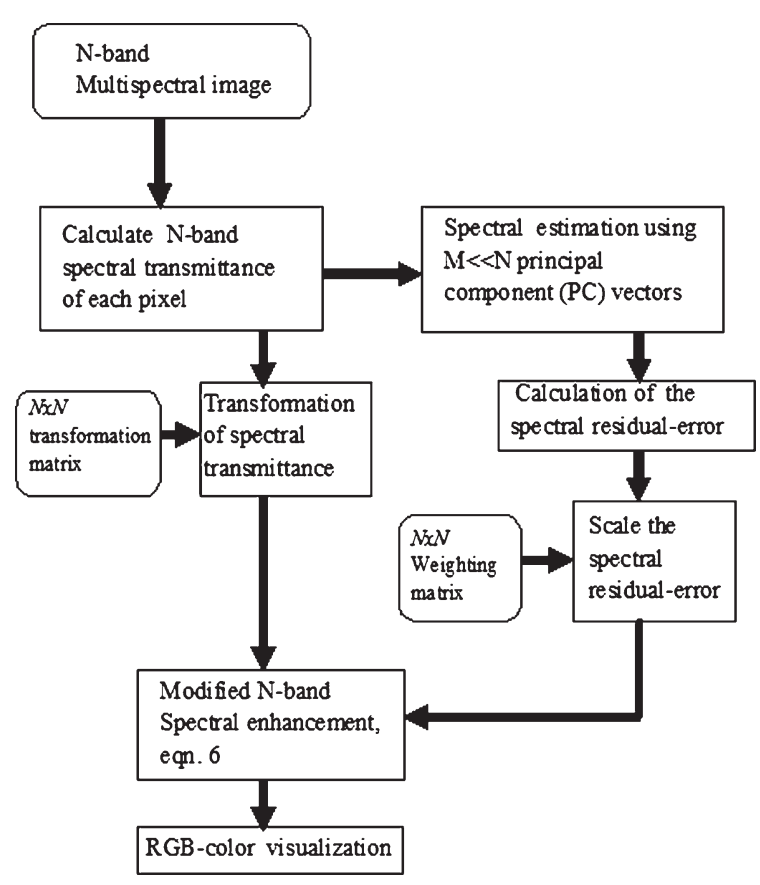

Fig. 1. The proposed multispectral enhancement method.

transformation matrix, $\mathbf{Q}$ into Eqn. 1 as follows:

$$
\mathbf{t}_{\mathrm{e}}=\mathbf{Q t}_{\mathrm{o}}+\mathbf{W \varepsilon}
$$

The $N \times N$ matrices $\mathbf{W}$ and $\mathbf{Q}$ respectively control the color of the objects of interest and the background pixels. Equation 6 generates the same result as eqn. 1 when $\mathbf{Q}$ is set to a diagonal matrix. The components of $\mathbf{Q}$ can be estimated using the method described in [3], i.e., using least mean square approach. Figure 1 illustrates the block diagram of the present multispectral enhancement.

\section{Results}

\subsection{Spectral residual-errors}

Spectral transmittance samples of the background objects or of those tissue components which are not subject for enhancement were used to derive the principal component (PC) vectors. The spectral residual errors were then determined by subtracting the estimated transmittance calculated using $m \ll N$ PC vectors from the original transmittance.

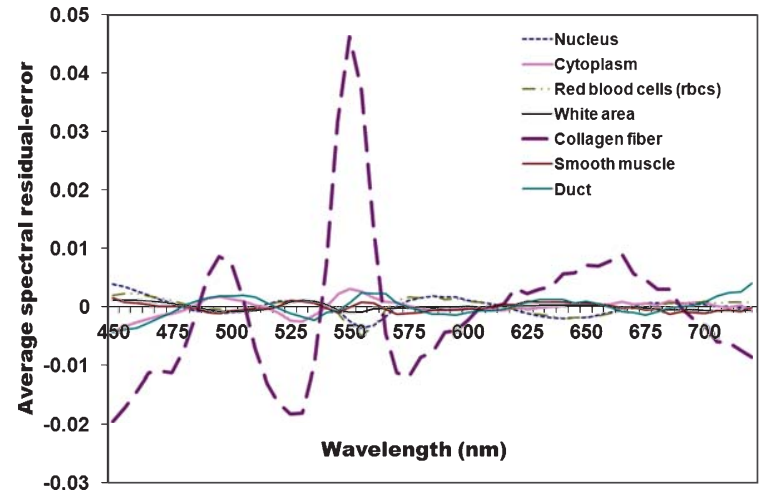

Fig. 2. Spectral residual-error of the different tissue components. Around $550 \mathrm{~nm}$ the spectral-error difference between the collagen fiber, which is the object of interest, and the other tissue components is large.

\subsection{Band for enhancement, $n$}

The band for enhancement was identified from the spectral residual-error configuration of the collagen fiber, which was the object of interest. Transmittance samples of the collagen fiber were extracted from the H\&E stained multispectral images and their spectral errors were calculated. Figure 2 shows the average spectral residual-error of the collagen fiber and of the tissue components which were considered as background objects. The band for enhancement corresponds to the wavelength at which the error of the collagen fiber exhibit significant peak. From the figure we can observe that at around $550 \mathrm{~nm}$ the collagen fiber acquired significantly larger error than the other tissue components. Hence, the band for enhancement, $n$, was set to $550 \mathrm{~nm}$.

\subsection{Spectral-color for enhancement}

The target spectral-color for enhancement was specified by the user. In the present experiment, we used the spectral color of a Masson's trichrome stained collagen-fiber as the target spectrum. Figure 3 shows the original H\&E and Masson's trichrome stained transmittance of the collagen fiber.

\section{4. $N \times N$ transformation matrix $Q$}

The transformation matrix Q was derived following the procedure outlined in [3]. The spectral data set consisted of the H\&E and Masson's trichrome spectra of 


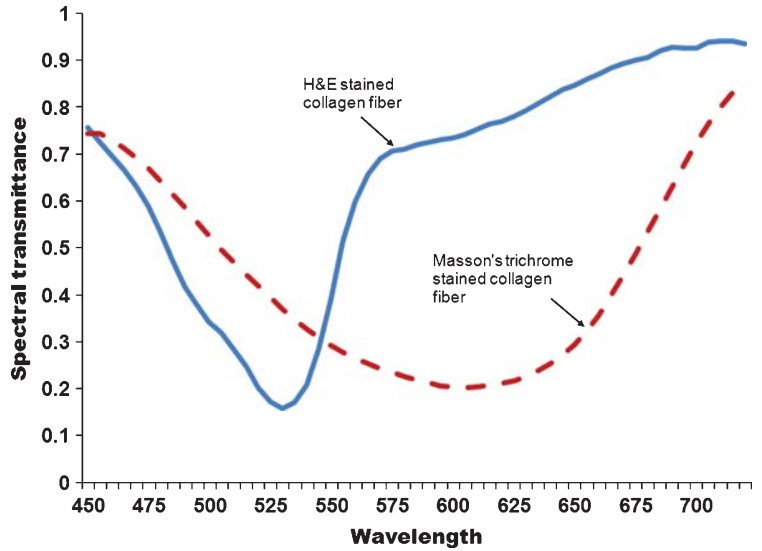

Fig. 3. Illustration of the collagen fiber spectral transmittance configuration when stained with H\&E and Masson's trichrome stain.

the background objects. That is, the spectral transmittance samples of the collagen fiber were excluded.

\subsection{Enhanced multispectral image visualization}

Visualization of multispectral image can be done by converting the pixel's $N$-band spectral signals to their RGB signals [3]. The images in the first and second rows in Fig. 4 illustrate the H\&E and Masson's trichrome stained RGB- images, respectively. We can see that it is easier to discriminate the collagen fibers from the Masson's trichrome stained images rather than from the H\&E stained images. The original H\&E stained images were enhanced using eqn. 6 and the results are show in Fig. 5. The original colors of the pixels were not only changed, but they were changed according to the association of the tissue structures to Masson's trichrome stain, Fig. 4.

\section{Conclusion}

A popular approach to visualizing objects in a multispectral image is to apply principal component analysis (PCA) wherein the images corresponding to the first three dominant components are mapped to RGB (reg, green and blue) or HSV (hue, saturation and value) $[6,7]$. Independent component analysis (ICA) has also been adopted for multispectral enhancement to improve the visualization of biological cells [8]. While these approaches have the capability to visualize multispectral features compactly, they don't render
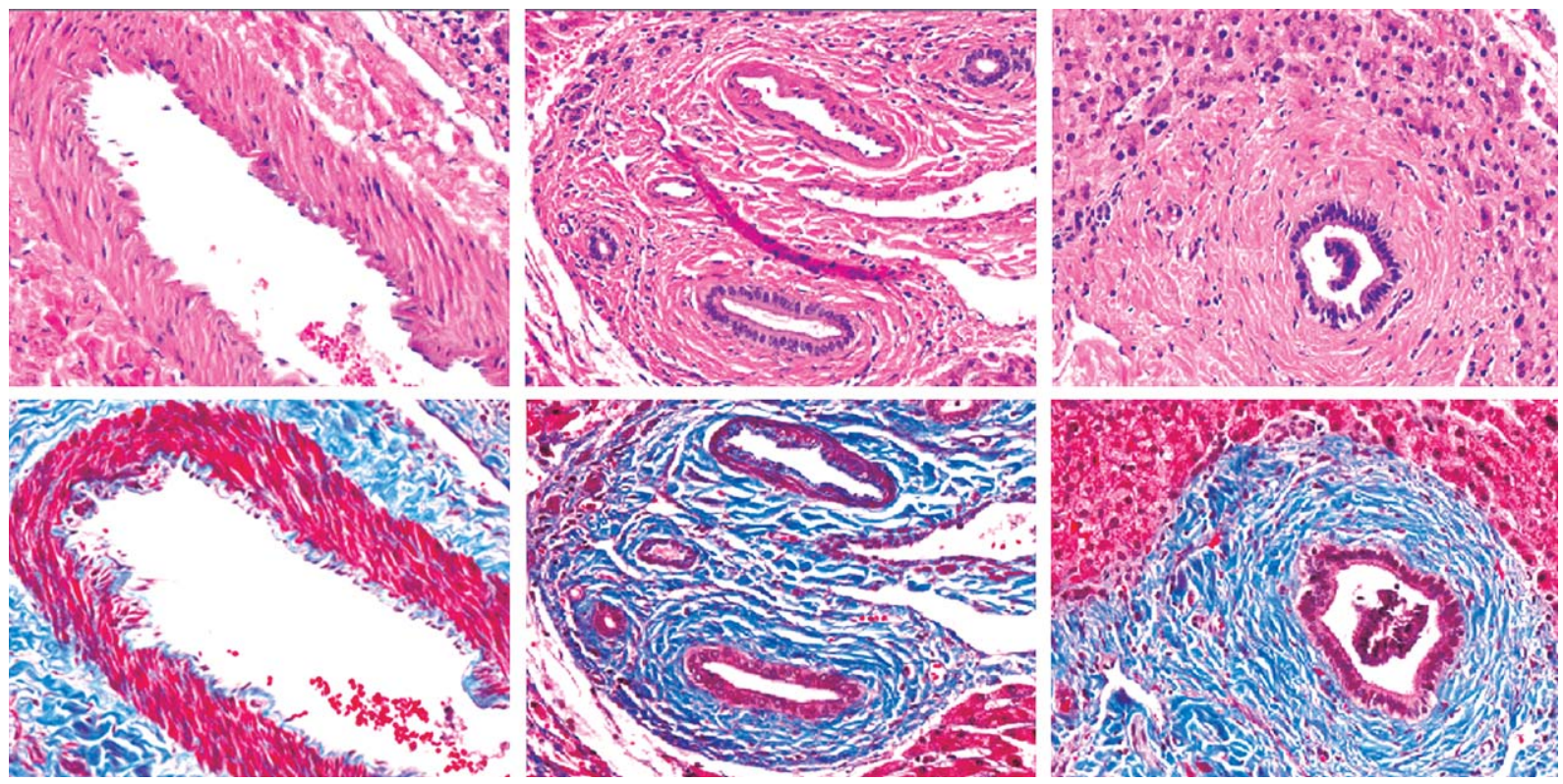

Fig. 4. The RGB-color images of the H\&E and Masson's trichrome image samples. These images show how staining improves the visualization of certain tissue structures. From the Masson's trichrome stained images we can clearly see the difference between the collagen fiber and non-collagen fiber. 

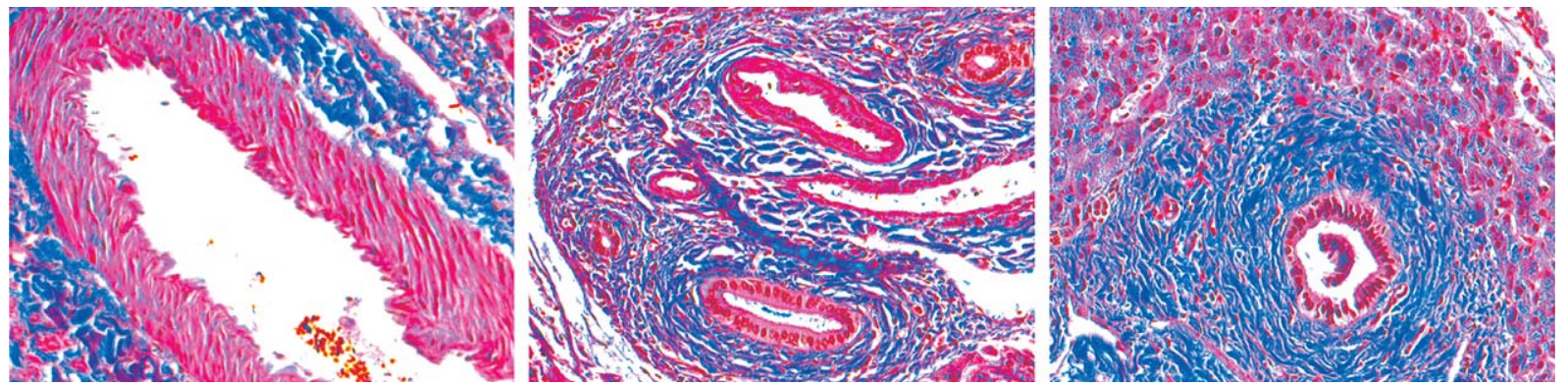

Fig. 5. Results of the proposed multispectral enhancement method. We can see that compared to their original H\&E stained images in Fig. 4, the collagen fibers could be easily discriminated from the rest of the eosin (E) stained structures. Also, the acquired colors of the pixels are closely similar to the Masson's trichrome staining patterns, Fig. 4. These results show the potential of the proposed enhancement method to implement digital staining.

consistent color for display. That is, the visualization color varies when the image content varies. In contrast, the multispectral enhancement methods proposed in $[4,5]$ assigns similar color to the objects of interest regardless of the image content. Consistent color rendering of the enhanced multispectral features is advantageous - it enables users to learn the relation between the enhanced spectral-color and the object of interest.

In this paper, we introduced a modification to the enhancement methods in $[4,5]$ to encompass the concept of digital staining wherein the color changes are aligned to objects' reactions to the target chemical stain. The experimental results show the potential of the present multispectral enhancement scheme to simulate the colorization effect of physical staining- digital staining, particularly the digital conversion of an $\mathrm{H} \& \mathrm{E}$ to Masson's trichrome stained image. Since the color impressions of digitally stained images are familiar to pathologists it will be easy for them to identify the objects of interest. With the effective and efficient implementation of digital staining, the cost of staining can be reduced while delivering diagnostic results at a shorter time.

\section{References}

[1] Sobota/Hammersen, Histology Color Atlas of Microscopic Anatomy, 3rd edn., Urban and Schwarzenberg, BaltimoreMunich, (1985).

[2] P.A. Bautista, T. Abe, M. Yamaguchi and N. Ohyama, Digital staining for multispectral images of pathological tissue specimens based on combined classification of spectral transmittance, Comp Med Imaging and Graphics 29 (2005), 649-657.

[3] P.A. Bautista and Y. Yagi, Digital staining for histopathology images by the combined application of spectral enhancement and spectral transformation, Proc IEEE EMBC, Boston (2012), 8013-8016.

[4] N. Hashimoto, Y. Murakami, P.A. Bautista, et al., Multispectral image enhancement for improve visualization, Optics Express 19 (2011), 9315-9329.

[5] M. Mitsui, Y. Murakami, T. Obi, M. Yamaguchi and N. Ohyama, Color enhancement in multispectral image using KarhunenLoeve transform, Opt Rev 12 (2005), 69-75.

[6] J.S. Tyo, A. Konsolakis, D.I. Diersen and R.C. Olsen, "Principalcomponents-based display strategy for spectral imagery," IEEE Trans, Geosci Rem Sens 41 (2003), 708-718.

[7] N.P. Jacobson and M.R. Gupta, Designs goals and solutions for display of hyperspectral images, IEEE Trans On Geoscience and Remote Sensing 43 (2003), 2684-2694.

[8] L.L. Nuffer, P.A. Medveck, H.P. Foote and J.C. Solinsky, Multispectral/hyperspectral image enhancement for biological cell analysis, Cytometry Part A 60A (2006), 897-903. 


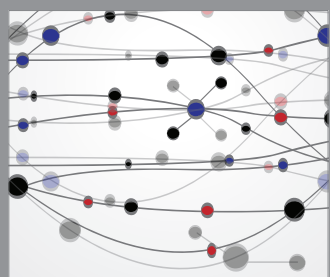

The Scientific World Journal
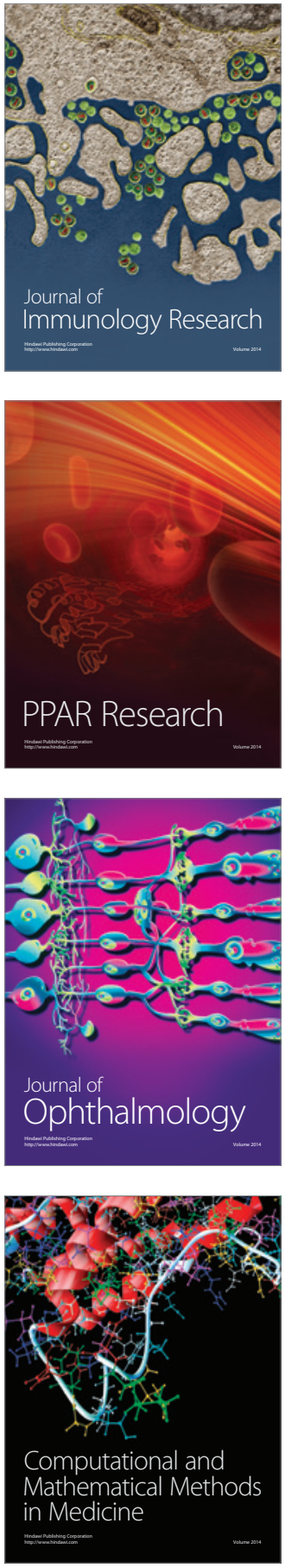

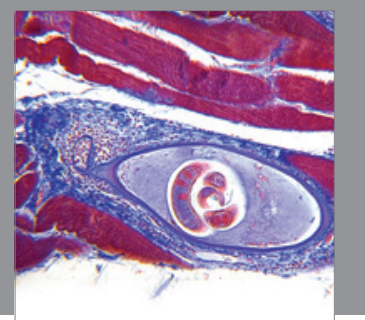

Gastroenterology

Research and Practice
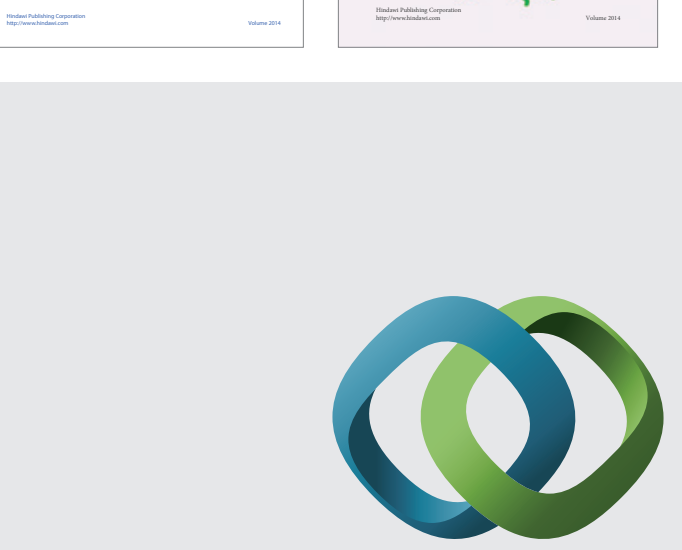

\section{Hindawi}

Submit your manuscripts at

http://www.hindawi.com
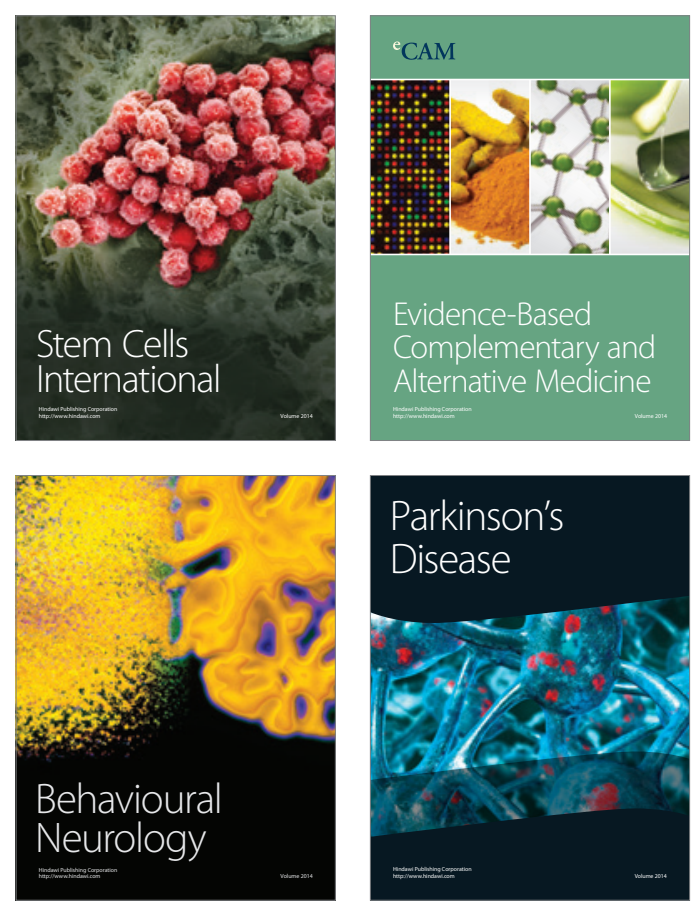

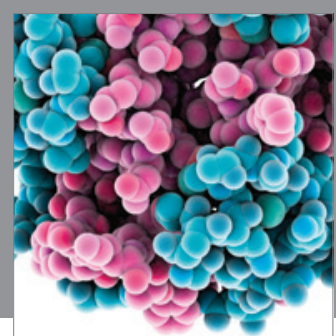

Journal of
Diabetes Research

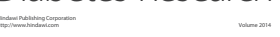

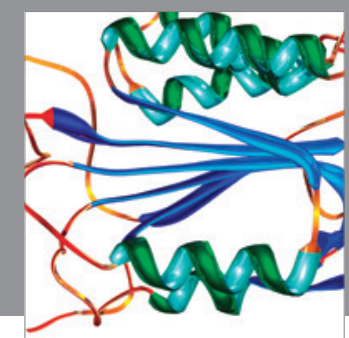

Disease Markers
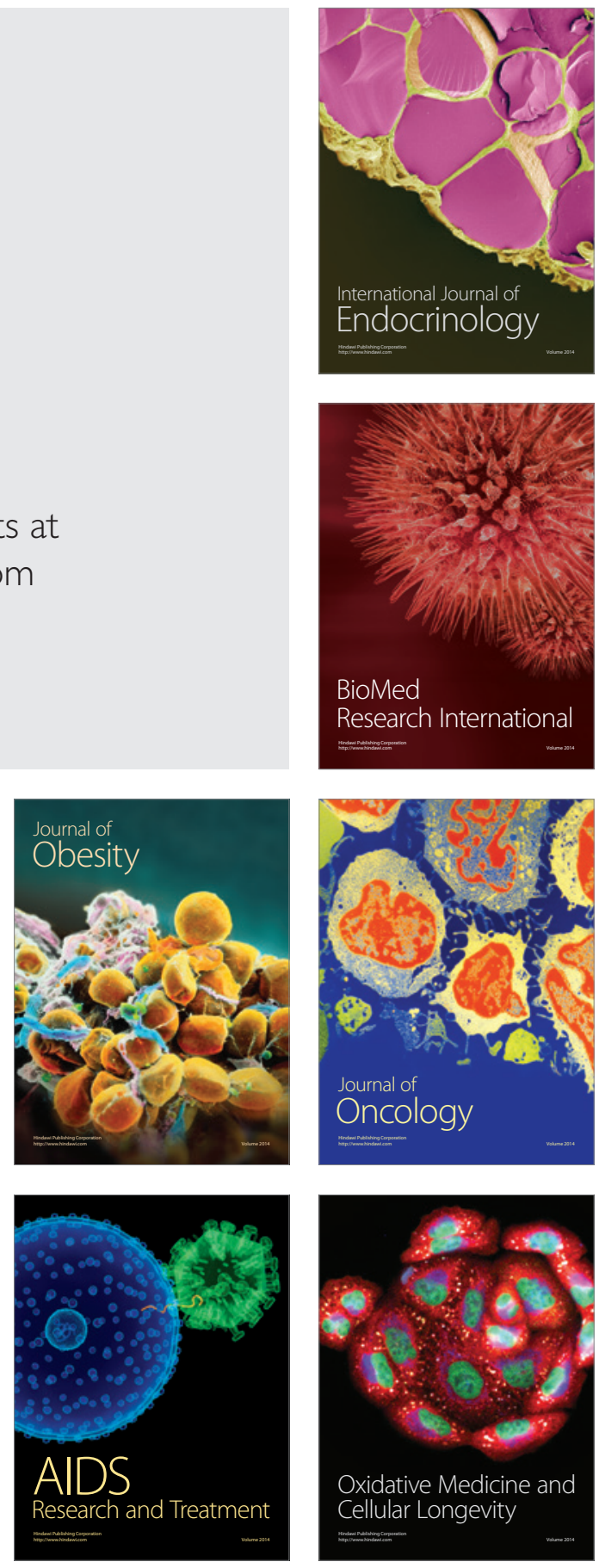